\title{
EXISTENTIALLY CLOSED MODELS IN THE FRAMEWORK OF ARITHMETIC
}

\author{
ZOFIA ADAMOWICZ, ANDRÉS CORDÓN-FRANCO, AND F. FÉLIX LARA-MARTÍN
}

\begin{abstract}
We prove that the standard cut is definable in each existentially closed model of $I \Delta_{0}+\exp$ by a (parameter free) $\Pi_{1}$-formula. This definition is optimal with respect to quantifier complexity and allows us to improve some previously known results on existentially closed models of fragments of arithmetic.
\end{abstract}

1. Introduction. This work was initially motivated by a gap in the proof of Corollary 1.3 of [2] providing a parameter free $\Pi_{1}-$ definition of the standard cut, $\mathbb{N}$, in each existentially closed (e.c.) model of $I \Delta_{0}+$ exp. Our aim is to provide a correct proof of the above result and, use it to obtain an updated view of the theory of e.c. models of $I \Delta_{0}+$ exp.

Existentially closed models of arithmetic were investigated in the 1970's as a part of the efforts to get a full understanding of the model theory of existentially closed structures (existence of model completions and companion theories, finite and infinite forcing, etc.). The results obtained in the early 1970's by A. Robinson, J. Hirschfeld, D. C. Goldrei, A. Macintyre, and H. Simmons pointed out the most important property of e.c. models of sufficiently strong arithmetic theories: there exist formulas defining $\mathbb{N}$ in each such model. These results were not stated in their full generality. In the 1970's a systematic study of fragments of Peano arithmetic $P A$ was still to come and the authors focused essentially on e.c. models of $\Pi_{2}(\mathbb{N})$ (the set of true $\Pi_{2}$-sentences) or of $\Pi_{2}(P A)$ (the set of $\Pi_{2}$ consequences of $P A$ ), and more generally on e.c. models of $\Pi_{2}\left(T_{B}\right)$, where $T_{B}$ is any extension of $\Pi_{2}(P A)$.

Regarding $\Pi_{2}(\mathbb{N})$, Robinson (see [14]) proved $\mathbb{N}$ to be $\Sigma_{3}$-definable in every e.c. model of $\Pi_{2}(\mathbb{N})$ and Hirschfeld (see [7]) improved Robinson's result obtaining a $\Sigma_{2}$-definition of $\mathbb{N}$, or even a $\Pi_{1}$-definition, if parameters are allowed. Hirschfeld also showed that these definitions are optimal (in terms of quantifier complexity) for e.c. models of $\Pi_{2}(\mathbb{N})$.

As to $\Pi_{2}\left(T_{B}\right)$, in [11] Macintyre and Simmons (see also [5]) extended Hirschfeld's $\Sigma_{2}$-definition of $\mathbb{N}$ to all e.c. models of $\Pi_{2}\left(T_{B}\right)$ and showed that the parametric $\Pi_{1}-$ definition can be extended to those e.c. models of $\Pi_{2}\left(T_{B}\right)$ in which the $\Sigma_{1}$-definable elements are not cofinal. However, these definitions are not best possible, since there is no general result ruling out the possibility of a parameter free $\Pi_{1}$-definition of $\mathbb{N}$ valid in all e.c. models. As a matter of fact, such an optimal definition was

Key words and phrases. fragments of Peano arithmetic, existentially closed models, turing degrees of arithmetic theories. 
obtained by K. McAloon in [12] for recursively axiomatized extensions of $\Pi_{2}(P A)$, introducing new techniques in this topic. While previous definitions of $\mathbb{N}$ were obtained using tools from Computability Theory (creative sets in [5] and simple sets in [7] and [11]) McAloon used consistency statements to produce his definition.

It is natural to ask ourselves whether these results can be extended to weaker theories, ideally, to every extension of $I \Delta_{0}$. In fact, e.c. models of $I \Delta_{0}$ (or more precisely $\Sigma_{1}$-closed models, a tightly related notion defined in Section 2 ) are connected to notorious open problems in weak arithmetics. Remarkably, deciding whether a $\Sigma_{1}$-closed model of $I \Delta_{0}$ can satisfy the $\Sigma_{1}$-collection scheme $B \Sigma_{1}$ would have important consequences: a positive answer would solve (in the negative) the End Extension Problem; whereas a negative answer would provide us with a model without $\Sigma_{1}$-collection and without exponentiation. This points out the interest of obtaining a better understanding of e.c. models of $I \Delta_{0}$ but also suggests that this goal could be out of reach of current methods (see [3] and [4] for more information).

The situation is more satisfactory in the case of moderately weak theories proving that exponentiation is total. Let $T_{E}$ denote a recursively axiomatized extension of $I \Delta_{0}+$ exp. It follows from Simmons' remarks in [16] that the definitions of $\mathbb{N}$ given in [11] apply equally well to e.c. models of $\Pi_{2}\left(T_{E}\right)$. In contrast, McAloon's optimal $\Pi_{1}$-definition seems to be valid only for theories extending $P A$, as its proof uses the Arithmetized Completeness Theorem and, more importantly, requires $T_{E}$ to be a reflexive theory. In [2] a parameter free $\Pi_{1}$-definition of $\mathbb{N}$ valid in each e.c. model of $\Pi_{2}\left(T_{E}\right)$ was proposed. This definition is also based on a consistency notion (introduced by H. Kotlarski in [9]) and avoids the use of the Arithmetized Completeness Theorem. But, unfortunately, there is a gap in the proof of Corollary 1.3 of [2] stating the correctness of such a definition. The proposed definition can be shown to be correct if some form of reflection is provable in $T_{E}$ (see comments in Section 3) but this seems to require $T_{E}$ to be an extension of $P A$ again.

Here, and this is the main result of the paper, we give a corrected version of Corollary 1.3 of [2]. By using a new restricted consistency notion different from Kotlarski's, we show $\mathbb{N}$ to be parameter free $\Pi_{1}$-definable in each e.c. model of $\Pi_{2}\left(T_{E}\right)$ (see Theorem 3.4). Thus, we extend McAloon's result to every recursive extension of $I \Delta_{0}+\exp$ and provide an optimal definition of $\mathbb{N}$ in each e.c. model of such theories. As an application, we use this optimal definition to get answers to some questions on e.c. models of arithmetic left over in [16].

The paper is organized as follows. In Section 2 we introduce the notion of a $\Sigma_{1}$-closed model (which coincides with the notion of an e.c. model when $T$ extends $I \Delta_{0}+\exp$ ) and state its basic properties. We also discuss some simple applications of consistency statements (the basic technique of this paper) to the study of $\Sigma_{1}$-closed models. In Section 3, we prove the main result, Theorem 3.4, discuss some natural generalizations of it, and show that the parameter free $\Pi_{1}$-definition given by that theorem is optimal. The rest of the paper is devoted to applications of the obtained results. In Section 4, we deal with recursive saturation and the distribution of definable elements in $\Sigma_{1}$-closed models, answering two questions posed by Simmons in [16]. Finally, in Section 5 we characterize the Turing degrees of maximal theories.

2. $\Sigma_{1}$-closed models and consistency statements. In this paper we shall work with a variant of the notion of an existentially closed model of a theory 
$T: \Sigma_{1}-$ closed models. Although for theories extending $I \Delta_{0}+\exp$ both notions coincide $\left(I \Delta_{0}+\right.$ exp proves Matiyasevich's theorem), they might differ for plain $I \Delta_{0}$ or other theories below exp. Let us give the precise definition. We assume familiarity with basic notions and results concerning fragments of arithmetic (all relevant information can be found in [6] and [8]). We denote by $\mathcal{L}$ the usual language of first order arithmetic $\{0,1,+, \cdot,<\}$. If $M$ and $N$ are $\mathcal{L}$-structures, we write $M \prec_{n} N$ if $M$ is a substructure of $N$ and, for all $\varphi\left(x_{1}, \ldots, x_{m}\right) \in \Sigma_{n}$ and all $a_{1}, \ldots, a_{m} \in M$, $M \models \varphi\left(a_{1}, \ldots, a_{m}\right) \Leftrightarrow N \models \varphi\left(a_{1}, \ldots, a_{m}\right)$.

Definition 2.1. Let $T$ be an extension of $I \Delta_{0}$. An $\mathcal{L}$-structure $M$ is a $\Sigma_{1}$-closed model of $T$ if $M \models T$ and for each $N \models T$, we have: $M \prec_{0} N \Rightarrow M \prec_{1} N$.

It is worth noting that a structure $\Sigma_{1}$-closed with respect to one theory may not be $\Sigma_{1}$-closed with respect to another one. However, often we will simply write 'let $M \models T$ be $\Sigma_{1}$-closed.' This should be seen as a shorthand for 'let $M \models T$ be $\Sigma_{1}$-closed with respect to $T$.'

The basic model theoretic properties of $\Sigma_{1}$-closed models can be obtained, mutatis mutandis, as in the classical setting of existentially closed structures (see [15]):

Proposition 2.2. Let $M \models T$. The following are equivalent:

1. $M$ is a $\Sigma_{1}-$ closed model of $T$.

2. For each $\varphi(x) \in \Pi_{1}$ and each $a \in M$ such that $M \models \varphi(a)$, there exists $\theta(x) \in \Sigma_{1}$ such that $M \models \theta(a)$ and $T \vdash \forall x(\theta(x) \rightarrow \varphi(x))$.

3. $M$ is a $\Sigma_{1}$-closed model of $\Pi_{1}(T)$.

Proposition 2.3. Assume $I \Delta_{0} \subseteq T$.

1. If $M \models T$, there is $M \prec_{0} N$ such that $N$ is a $\Sigma_{1}$-closed model of $\Pi_{2}(T)$.

2. If $M$ is a $\Sigma_{1}$-closed model of $T$ and $N \prec_{1} M$, then $N$ is a $\Sigma_{1}$-closed model of $\Pi_{2}(T)$.

3. If $M$ and $N$ are $\Sigma_{1}$-closed models of $T$ and $M \prec_{0} N$, then $M \prec_{2} N$.

A useful corollary is that if $M$ is a $\Sigma_{1}$-closed model of $T$ and $X \subseteq M$, then $K(M, X)$ (i.e., the substructure of the elements which are $\Sigma_{1}$-definable in $M$ with parameters from $X)$ is a $\Sigma_{1}$-closed model of $\Pi_{2}(T)$ and so $K(M, X) \prec_{2} M$.

Note that the standard model $\mathbb{N}$ is $\Sigma_{1}$-closed with respect to a theory $T$ if, and only if, $\mathbb{N} \models T$ and $T$ implies $\Pi_{1}(\mathbb{N})$. As to how much arithmetic a nonstandard $\Sigma_{1}$-closed model can satisfy, it is known that $\Sigma_{1}$-closed models do not exist even for moderately weak fragments of $P A$.

Proposition 2.4. Assume $I \Delta_{0} \subseteq T$. Let $M \models T$ be $\Sigma_{1}$-closed.

1. If $M$ is nonstandard, $M \not \models B \Sigma_{1}+\exp$.

2. If $T$ is recursively axiomatized, $M \forall \forall I \Pi_{1}^{-}$and $M \not \models L \Delta_{1}^{-}+\exp$.

Proof. We only prove part (1), for part (2) see [4]. Let $a \in M$ nonstandard. Then $K(M, a) \prec_{2} M$. Thus, $M \not \models B \Sigma_{1}+\exp$, for it is well known that $K(M, a) \forall$ $B \Sigma_{1}+\exp$ and this last theory is $\Pi_{3}$-axiomatizable.

The consistency assertion for $T, \operatorname{Con}(T)$, is well known to fail in every $\Sigma_{1-}$ closed model of $T$ provided $T$ is a sufficiently strong, recursive theory (see, e.g., Theorem 1.1 of [2]). We include a proof of this fundamental fact, which is a simplification of that of [2]. As usual, $\operatorname{Prov}_{T}(x)$ denotes the standard provability predicate for $T$. 
Proposition 2.5. Let $T$ be a recursive extension of $I \Delta_{0}+\exp$ and let $M \models T$ be $\Sigma_{1}$-closed. Then

1. ( $\Pi_{1}$-completeness) For each $\varphi(x) \in \Pi_{1}, M \models \forall x\left(\varphi(x) \rightarrow \operatorname{Prov}_{T}(\varphi(\dot{x}))\right)$.

2. $M \models \neg \operatorname{Con}(T)$.

ProOF.

(1) Assume $M \models \varphi(a)$, with $\varphi(x) \in \Pi_{1}$. Since $M$ is $\Sigma_{1}$-closed, there is $\theta(x) \in \Sigma_{1}$ such that $M \models \theta(a)$ and $T \vdash \forall x(\theta(x) \rightarrow \varphi(x))$. By provable $\Sigma_{1}$-completeness, $M \models \operatorname{Prov}_{T}(\theta(\dot{a}))$ and so $M \models \operatorname{Prov}_{T}(\varphi(\dot{a}))$.

(2) Towards a contradiction, assume $M \models \operatorname{Con}(T)$. Then, for each sentence $\varphi \in \Pi_{1}, M \models \varphi \leftrightarrow \operatorname{Prov}_{T}(\varphi)$. (The implication from left to right follows by part (1) and the converse follows by provable $\Sigma_{1}$-completeness.) This gives us a $\Sigma_{1}$-definition of $\Pi_{1}$-truth in $M$, contradicting Tarski's theorem.

The above argument illustrates one of the main themes in this work: the use of consistency statements to derive properties of $\Sigma_{1}$-closed models. This idea can also be applied to $\Sigma_{1}$-closed models of theories below exp at the price of considering some restricted provability notion. As a prominent example, we include a proof of Theorem 2 of [1] (proved there by using a model-theoretic construction).

Theorem 2.6. Let $M$ be a $\Sigma_{1}$-closed model of I $\Delta_{0}$. Then $M \not \models \exp$.

Proof. Suppose $M \models$ exp. We shall derive a contradiction as in the proof of Proposition 2.5 but we replace the standard provability predicate by $\mathrm{Cut}$ free provability, (see [6], Chapter V, Section 5-(d) for details). Let $\operatorname{RPr}_{I \Delta_{0}}(u, x)$ denote a $\Sigma_{1}$ formula expressing that "there exists a conjunction, $c$, of axioms of $I \Delta_{0}$ (including equality axioms) and a proof of $c \rightarrow x$ (in the version of Schwichtenberg's calculus used in [6]) with cut-rank bounded by $u$." In addition, $\mathrm{CFPr}_{I \Delta_{0}}(x)$ denotes the formula $\operatorname{RPr}_{I \Delta_{0}}(0, x)$, and CFCon ${ }_{I \Delta_{0}}$ denotes the sentence $\neg \operatorname{CFPr}_{I \Delta_{0}}(0=1)$.

We shall show that for each $\Pi_{1}$ sentence, $\varphi, M \models \varphi \leftrightarrow \operatorname{CFPr}_{I \Delta_{0}}(\varphi)$, giving the desired contradiction.

First, assume $M \models \operatorname{CFPr}_{I \Delta_{0}}(\varphi)$ and $M \models \neg \varphi$. By formalized $\Sigma_{1}$-completeness (Lemma 5.24 in [6]), $M=\operatorname{RPr}_{I \Delta_{0}}(k, \neg \varphi)$ for some $k \in \omega$. Using formalized cut elimination (available in $I \Delta_{0}+\exp$, see Theorem 5.17-(ii) in [6]) we obtain that $M \models \operatorname{CFPr}_{I \Delta_{0}}(\neg \varphi)$. Then, $M \models \neg \operatorname{CFCon}_{I \Delta_{0}}$. But this is impossible since $I \Delta_{0}+\exp$ proves $\mathrm{CFCon}_{I \Delta_{0}}$ (see [17]).

Conversely, assume $M \models \varphi$. Since $M$ is $\Sigma_{1}$-closed, there is a sentence $\theta \in \Sigma_{1}$ such that $M=\theta$ and $I \Delta_{0} \vdash \theta \rightarrow \varphi$. Then, $M \models \operatorname{CFPr}_{I \Delta_{0}}(\neg \theta \vee \varphi)$. Since $M=\theta$, by formalized $\Sigma_{1}$-completeness, there is $k^{\prime} \in \omega$ such that $M \models \operatorname{RPr}_{I \Delta_{0}}\left(k^{\prime}, \theta\right)$. Using formalized cut elimination, we get $M \models \operatorname{CFPr}_{I \Delta_{0}}(\theta \vee \varphi)$. So, $M \models \operatorname{CFPr}_{I \Delta_{0}}(\varphi)$.

3. Defining the standard cut in $\Sigma_{1}$-closed models. In this section we present a corrected version of Corollary 1.3 of [2] and thus obtain an (optimal) definition of $\mathbb{N}$ in every $\Sigma_{1}$-closed model of a recursively axiomatized extension of $I \Delta_{0}+$ exp. Before doing so, let us give the exact statement of Corollary 1.3 in [2] and explain why that result is problematic. 
"Assume $T \subseteq \hat{T}$ and $T \supseteq I \Delta_{0}+\exp$. Let $M \models T$ be $\Sigma_{1}$-closed. Then for $v \in M, v \notin \mathbb{N}$ iff $M \models \neg \operatorname{Cons}_{K}(\hat{T}, v), "$

where $\hat{T}$ denotes a recursive and consistent extension of $I \Delta_{0}+\exp$ and the formula $\operatorname{Cons}_{K}(\hat{T}, v)$ denotes a restricted notion of consistency introduced by $\mathrm{H}$. Kotlarski in [9]. Namely, if we put $Q_{0}=\Delta_{0}$ and $Q_{n+1}=$ the closure of $Q_{n} \cup \exists Q_{n}$ under conjunction, negation and bounded quantification, then $\operatorname{Cons}_{K}(\hat{T}, v)$ denotes a $\Pi_{1}$ formula expressing that there is no proof of $0=1$ in $\hat{T}$ which consists of $Q_{v}$-formulas only.

First, it should be noted that the implication " $v \notin \mathbb{N} \Rightarrow M \models \neg \operatorname{Cons}_{K}(\hat{T}, v)$ " is true (this is Theorem 1.2 in [2]). But for the opposite implication to be true a necessary condition on $M$ is missing.

Lemma 3.1. If $M \models T$ and $M=\operatorname{Cons}_{K}(\hat{T}, n)$ for all $n \in \mathbb{N}, M \models \Pi_{1}(\hat{T})$.

Proof. Consider $\varphi \in \Pi_{1}(\hat{T})$. Let $\operatorname{Pr}_{K, \hat{T}}(x, v)$ express that there is a proof of $x$ in $\hat{T}$ included in $Q_{v}$. Then, $M \models \operatorname{Pr}_{K, \hat{T}}(\varphi, k)$ for some standard $k$. Now assume $M \forall \models \varphi$. By provable $\Sigma_{1}$-completeness (see remark 1.3 in [2]), $M \models P r_{K, \hat{T}}(\neg \varphi, 1)$. But then $M \models \neg \operatorname{Cons}_{K}(\hat{T}, k)$, a contradiction.

This first problem can be easily solved. Either we add the assumption $M \models \Pi_{1}(\hat{T})$ or, for the sake of simplicity, we take $T=\hat{T}$. Nevertheless, a second problem arises. The implication " $M \models \neg \operatorname{Cons}_{K}(\hat{T}, v) \Rightarrow v \notin \mathbb{N}$ " is not justified unless we assume $\hat{T} \supset P A$. In fact, this implication follows from Lemma 3 (on consistency)

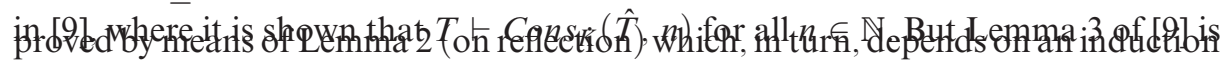
argument involving a formula in $Q_{n+1}$. So Lemma 3 of [9] is valid only under the assumption that $\hat{T} \supseteq P A$.

Therefore, Corollary 1.3 of [2] is true if we assume $M \models \Pi_{1}(\hat{T})$ and $\hat{T} \supseteq P A$, thus giving a new proof of McAloon's result on $\Pi_{1}$-definability of $\mathbb{N}$ in every e.c. model of $\Pi_{2}(P A)$ by using Kotlarski's consistency notion. But the general version stated in [2] for each $\hat{T}$ extending $I \Delta_{0}+\exp$ is unjustified.

Nevertheless, and this is the main result of the present paper, it is possible to get round this obstacle and obtain a corrected version of Corollary 1.3 of [2] by introducing a different restricted consistency notion.

Given a model $M$, for each $b \in M, C_{b}$ denotes the set of $\Sigma_{1}$ sentences $\phi\left(\tilde{a}_{1}, \ldots, \tilde{a} u\right)$, where, for $a \in M, \tilde{a}$ denotes the $a$-th numeral and $\phi\left(x_{1}, \ldots, x_{u}\right)$ is a formula (in the sense of $M$ ) with $x_{1}, \ldots, x_{u}$ as free variables and such that $\phi\left(x_{1}, \ldots, x_{u}\right) \leq b$. In addition, $D_{b}$ denotes the set of all formulas which are of the form $\phi\left(\tilde{a}_{1}, \ldots, \tilde{a}\right)$, where $\tilde{a} \tilde{1}_{1}, \ldots \dot{a}{ }_{u}$ are some numerals and $\phi\left(x_{1}, \ldots, x_{u}\right) \leq b$ (note that formulas from $D_{b}$ may contain other free variables than those which are explicitly indicated).

Let $\operatorname{Tr}_{n}$ be the usual truth definition for $\Sigma_{n}$ sentences given in $I \Delta_{0}+$ exp. Let $\operatorname{Pr}_{S}(\varphi, v)$ express the meaning that there is a proof of $\varphi$ (in the theory $S$ ) included in $D_{v}$ whose length is at most $v$, and let $\tilde{C}$ ons $(S, v)$ be the formula $\neg \operatorname{Pr}_{S}(\tilde{0}=\tilde{1}, v)$. Then, the formula $\operatorname{Cons}(\hat{T}, v)$ is

$$
\forall \phi \in C_{v}\left(\operatorname{Tr}_{1}(\phi) \rightarrow \tilde{C} \text { ons }\left(T^{\wedge}+\phi, v\right)\right) .
$$


That is to say, Cons $(\hat{T}, v)$ expresses the meaning that $\hat{T}$ is " $v$-consistent" (in the sense $\tilde{C}$ ons) with any $\Sigma_{1}$ true sentence. Now the corrected version of Corollary 1.3 in [2] is:

"Assume $\hat{T}$ is recursive and $I \Delta_{0}+\exp \subseteq T \subseteq \hat{T}$. Let $M \models T$ be $\Sigma_{1}$-closed.

Assume $M \models \Pi_{1}(\hat{T})$. Then for $b \in M, b \notin \mathbb{N}$ iff $M \models \neg \operatorname{Cons}(\hat{T}, b)$."

In what follows we give a proof of this result (see Theorem 3.4). We first prove the implication from left to right.

Proposition 3.2. Assume $\hat{T}$ is recursive and $I \Delta_{0}+\exp \subseteq T \subseteq \hat{T}$. Let $M \models T$ be $\Sigma_{1}-$ closed. Then for every nonstandard $b \in M, M \models \neg \operatorname{Cons}(\hat{T}, \bar{b})$.

Proof. We repeat the proof of Theorem 1.2 in [2] with suitable modifications. By way of contradiction, let $b \in M$ be nonstandard and suppose that $M \models \operatorname{Cons}(\hat{T}, b)$. Consider the following formula $\psi(\varphi, x, b)$ :

$$
\operatorname{Cons}(\hat{T}+\varphi(\tilde{x}, \tilde{b}), \sqrt{b}) .
$$

We shall show that $\psi$ is universal in $M$ for $\Sigma_{1}$ formulas with one free variable and the parameter $b$. That is, we shall show

$$
M \models \forall x(\psi(\varphi, x, b) \leftrightarrow \varphi(x, b)), \text { for } \varphi \in \Sigma_{1} .
$$

To prove this, first assume $M \models \varphi(a, b)$, for some $a \in M$. Let us show $M \models$ $\psi(\varphi, a, b)$. Note that $\varphi(x, v) \in \mathbb{N}$, whence $\varphi \leq \sqrt{b}$. Since $M \models \operatorname{Cons}(\hat{T}, b)$, we infer

$$
M \models \forall \phi \in C_{b}\left(\operatorname{Tr}_{1}(\phi) \rightarrow \tilde{C} \text { ons }(\hat{T}+\phi, b)\right) .
$$

Note that each $\phi \in C_{\sqrt{b}}$ is of the form $\theta\left(\tilde{a}_{1}, \ldots, \tilde{a}_{u}\right)$ for some $u, a_{1}, \ldots, a_{u} \in M$ and some $\Sigma_{1}$ formula $\theta\left(x_{1}, \ldots, x_{u}\right) \leq \sqrt{b}$. Therefore, from the fact that $\varphi \leq \sqrt{b}$, we infer that $\phi \wedge \varphi(\tilde{a}, \tilde{b})$ is in $C_{b}$ (we assume that our enumeration of formulas has the property that $\eta \wedge \zeta \leq \eta \cdot \zeta)$. Moreover, from the facts that $M=\varphi(a, b)$ and $M \models \operatorname{Tr}_{1}(\phi)$ we infer that $M \models \operatorname{Tr}_{1}(\phi \wedge \varphi(\tilde{a}, \tilde{b}))$. Then, in particular

$$
M \models \forall \phi \in C_{\sqrt{b}}\left(\operatorname{Tr}_{1}(\phi) \rightarrow \tilde{C} \text { ons }(\hat{T}+\phi \wedge \varphi(\tilde{a}, \tilde{b}), b)\right) .
$$

Hence

$$
M \models \forall \phi \in C_{\sqrt{b}}\left(\operatorname{Tr}_{1}(\phi) \rightarrow \tilde{C} \operatorname{ons}((\hat{T}+\varphi(\tilde{a}, \tilde{b}))+\phi, \sqrt{b})\right)
$$

for, if there was a proof of a contradiction using $\phi$ and $\varphi(\tilde{a}, \tilde{b})$ as premises which consists of instances of formulas $\leq \sqrt{b}$ and has length $\leq \sqrt{b}$, then there would be a proof of a contradiction using $\phi \wedge \varphi(\tilde{a}, \tilde{b})$ as a premise which would consist of instances of formulas $\leq b$ and would have length $\leq b$. Thus, we have $M \models \operatorname{Cons}(\hat{T}+\varphi(\tilde{a}, \tilde{b}), \sqrt{b})$. Hence $M \models \psi(\varphi, a, b)$.

Now assume that $M \models \psi(\varphi, a, b)$. We add to the language constants $\underline{u}$, for all $u \in M$, and consider the following theory $T^{\prime}$ in this extended language:

$T^{\prime}=\hat{T}+\left\{\theta\left(\underline{u}_{1}, \ldots, \underline{u}_{k}\right): \theta \in \Sigma_{1}, u_{1}, \ldots, u_{k} \in M, M \models \theta\left(u_{1}, \ldots, u_{k}\right)\right\}+\varphi(\underline{a}, \underline{b})$.

That is, $T^{\prime}$ is $\hat{T}$ plus the $\Sigma_{1}$ diagram of $M$ plus $\varphi(\underline{a}, \underline{b})$. We shall show that $T^{\prime}$ is consistent. To see this, consider a finite fragment $T^{\prime \prime} \subseteq T^{\prime}$ :

$$
\hat{T}^{\prime}+\left\{\theta_{1}\left(\underline{u}_{1,1}, \ldots, \underline{u}_{1, k_{1}}\right), \ldots, \theta_{n}\left(\underline{u}_{n, 1}, \ldots, \underline{u}_{n, k_{n}}\right)\right\}+\varphi(\underline{a}, \underline{b})
$$


(where $\hat{T}^{\prime}$ denotes a finite fragment of $\left.\hat{T}\right)$. By $\psi(\varphi, a, b)$ and since the formula $\theta_{1}\left(x_{1,1}, \ldots, x_{1, k_{1}}\right) \wedge \cdots \wedge \theta_{n}\left(x_{n, 1}, \ldots, x_{n, k_{n}}\right)$ (where each $x_{i, j}$ denotes a fresh variable) is a standard $\Sigma_{1}$ formula (and so $\leq \sqrt{b}$ ), we infer

$$
M \models \tilde{C} \text { ons }\left((\hat{T}+\varphi(\tilde{a}, \tilde{b}))+\theta_{1}\left(\tilde{u}_{1,1}, \ldots, \tilde{u}_{1, k_{1}}\right) \wedge \cdots \wedge \theta_{n}\left(\tilde{u}_{n, 1}, \ldots, \tilde{u}_{n, k_{n}}\right), \sqrt{b}\right) \text {. }
$$

Since $b$ is nonstandard, in particular, there is no standardly finite sequence of standard formulas which is a proof of contradiction from $T^{\prime \prime}$, since if there was such a sequence it would belong to $M$ (as a finite sequence of elements of $M$ ) and it would give a (standard) proof of contradiction from the theory

$$
\hat{T}+\varphi(\tilde{a}, \tilde{b})+\theta_{1}\left(\tilde{u}_{1,1}, \ldots, \tilde{u}_{1, k_{1}}\right) \wedge \cdots \wedge \theta_{n}\left(\tilde{u}_{n, 1}, \ldots, \tilde{u}_{n, k_{n}}\right)
$$

in $M$. Thus, $T^{\prime \prime}$ is consistent and, by compactness, $T^{\prime}$ is consistent.

Let $M^{\prime} \models T^{\prime}$. Then $M \prec_{0} M^{\prime}$ up to isomorphism since $T^{\prime}$ contains the $\Delta_{0}$ diagram of $M$. Moreover, since $\underline{a}$ is interpreted as $a$ and $\underline{b}$ as $b$, we infer that $M^{\prime} \models \varphi(a, b)$. Also, $M^{\prime} \models T$ since $T^{\prime} \supseteq \hat{T}$. Hence, by the fact that $M$ is $\Sigma_{1}$-closed, $M \models \varphi(a, b)$, which completes the proof of $(E)$.

Now, to complete the proof of the theorem, we repeat the reasoning of Theorem 1.1 in [2]: let $\varphi(x, b)$ the formula $\neg \psi(x, x, b)$. Then by $(E)$, taking $x=\varphi$, we have $\psi(\varphi, \varphi, b) \Leftrightarrow \varphi(\varphi, b) \Leftrightarrow \neg \psi(\varphi, \varphi, b)$, a contradiction.

In the proof of the opposite implication, we will have to restrict ourselves to certain special axiomatizations of $\hat{T}$. Namely, we say that $\varphi_{S}(x) \in \Delta_{1}$ is a wellbehaved presentation of a theory $S$ if: $i) \varphi_{S}(x)$ defines in $\mathbb{N}$ an axiomatization of $S$; and $i i)$ there is some standard $k$ such that $I \Delta_{0}+\exp$ proves the formula $\forall x\left(\varphi_{S}(x) \rightarrow \operatorname{Sent}(x) \wedge\right.$ "the numerals in $x$ are among $\tilde{0}, \ldots, \tilde{k}$ ").

LEMMA 3.3. Each recursive theory admits well-behaved presentations.

Proof. (Sketch) Let $S$ be a recursive theory. Let $S^{\prime}$ be the axiomatization of $S$ obtained by replacing each axiom of $S$ of the form $\forall x \theta(x, m)$ (we identify $m \in \mathbb{N}$ with its numeral) with

$$
\forall x \forall x_{0} \ldots \forall x_{m}\left(x_{0}=0 \wedge x_{1}=x_{0}+1 \wedge \cdots \wedge x_{m}=x_{m-1}+1 \rightarrow \theta\left(x, x_{m}\right)\right) .
$$

Then, $S^{\prime}$ is recursive in $S$ and so $S^{\prime}$ is recursive too. Let $\varphi_{S^{\prime}}(x) \in \Delta_{1}$ be a presentation of $S^{\prime}$. It suffices to put

$$
\varphi_{S}(x)=\varphi_{S^{\prime}}(x) \wedge \operatorname{Sent}(x) \wedge \text { "the numerals in } x \text { are among } \tilde{0}, \tilde{1} . "
$$

THeOREM 3.4. Assume $\hat{T}$ is recursive and $I \Delta_{0}+\exp \subseteq T \subseteq \hat{T}$. Let $M \models T$ be $\Sigma_{1}$-closed. Assume $M \models \Pi_{1}(\hat{T})$. Then, for each well-behaved presentation of $\hat{T}$ and for all $b \in M$,

$$
b \notin \mathbb{N} \text { iff } M \models \neg \operatorname{Cons}(\hat{T}, b) .
$$

Proof. The implication from left to right follows directly from Proposition 3.2. To see the implication from right to left, consider the contrapositive form:

$$
b \in \mathbb{N} \quad \Rightarrow \quad M \models \operatorname{Cons}(\hat{T}, b) .
$$

So, let $b \in \mathbb{N}$. Since $\operatorname{Cons}(\hat{T}, b) \in \Pi_{1}$ and $M=\Pi_{1}(\hat{T})$, it is sufficient to show that $\hat{T} \vdash \operatorname{Cons}(\hat{T}, b)$. To this end, let $M^{\prime} \models \hat{T}$ and let $\phi \in M^{\prime}$ such that $\phi \in C_{b}$ and $M^{\prime} \models \operatorname{Tr}_{1}(\phi)$. Let us show $M^{\prime} \models \tilde{C}$ ons $(\hat{T}+\phi, b)$. By way of contradiction, suppose that there is a proof from $\hat{T}+\phi$ of $\tilde{0}=\tilde{1}, d=\left\langle\psi^{0}, \ldots, \psi^{b-1}\right\rangle \in M^{\prime}$, 
such that for all $k<b, \psi^{k} \in D_{b}$. Since $b \in \mathbb{N}$, for each $k<b$ there exist a (standard) formula $\theta_{k}\left(x_{1}, \ldots, x_{r_{k}}\right)$ and elements $a_{k, 1}, \ldots, a_{k, r_{k}} \in M^{\prime}$ such that $\psi^{k}$ is the formula $\theta_{k}\left(\tilde{a}_{k, 1}, \ldots, \tilde{a}_{k, r_{k}}\right)$. Let us prove that $M^{\prime} \models \theta_{k}\left(a_{k, 1}, \ldots, a_{k, r_{k}}\right)$.

It is easy to check that if $\psi^{k}$ is a logical axiom then $\theta_{k}\left(x_{1}, \ldots, x_{r_{k}}\right)$ is also a logical axiom and, therefore, $M^{\prime} \models \theta_{k}\left(a_{k, 1}, \ldots, a_{k, r_{k}}\right)$. If $\psi^{k}$ is $\phi$, then $M^{\prime} \models$ $\operatorname{Tr}_{1}\left(\theta_{k}\left(\tilde{a}_{k, 1}, \ldots, \tilde{a}_{k, r_{k}}\right)\right)$ and, as a consequence, $M^{\prime}=\theta_{k}\left(a_{k, 1}, \ldots, a_{k, r_{k}}\right)$. Regarding the axioms of $\hat{T}$, since we are assuming that $\hat{T}$ is axiomatized by sentences not containing arbitrarily large numerals, if some $\psi^{k}$ in $d$ is an axiom of $\hat{T}$ then it must be a standard formula. Thus, $M^{\prime} \models \psi^{k}$ and so $M^{\prime} \models \theta_{k}\left(a_{k, 1}, \ldots, a_{k, r_{k}}\right)$. Then we can show by a straightforward induction on $k<b$ that $M^{\prime} \models \theta_{k}\left(a_{k, 1}, \ldots, a_{k, r_{k}}\right)$ (passing through modus ponens is evident). It follows that $M^{\prime}=0=1$, contradiction. $\dashv$

Taking $T=\hat{T}$, we obtain a simplified version of Theorem 3.4 that will suffice for applications.

COROLlary 3.5. Let $T$ be a recursive extension of $I \Delta_{0}+\exp$ and let $M \models T$ be $\Sigma_{1}$-closed. Then, for $b \in M, b \notin \mathbb{N}$ iff $M=\neg \operatorname{Cons}(T, b)$, for each well-behaved presentation of $T$.

Corollary 3.5 has a natural generalization to arbitrary, not necessarily recursively axiomatized extensions of $I \Delta_{0}+\exp$. Namely, assume $M$ is a $\Sigma_{1}$-closed model of $T$ in which $T$ is coded, i.e., there is some $c \in M$ such that the axioms of $T$ are given by $\left\{n \in \mathbb{N}: M \models(c)_{n} \neq 0\right\}$. We define the notion of a well-behaved code of $T$ in $M$ in the natural way. Reasoning as in the proof of Lemma 3.3, we get that $T$ also has a well-behaved code in $M$, say $a$. Then, we can construct the formula $\operatorname{Cons}(T, v, a)$ as above but now we use $a$ as a parameter in order to express the set of axioms of $T$. By repeating the proofs of Proposition 3.2 and Theorem 3.4, we get

Corollary 3.6. Suppose $I \Delta_{0}+\exp \subseteq T$. Let $M \models T$ be $\Sigma_{1}-$ closed with $T$ coded in $M$ and let $a \in M$ be a well-behaved code of $T$. Then, for $b \in M$,

$$
b \notin \mathbb{N} \quad \text { iff } \quad M \models \neg \operatorname{Cons}(T, b, a) .
$$

REMARK 3.7. In Theorem 8.29 of [7] Hirschfeld showed $\mathbb{N}$ to be $\Pi_{1}$-definable in each e.c. model of $\Pi_{2}(\mathbb{N})$. In Theorem 6.2 of [16] Simmons proved that if $M$ is an e.c. model of $T \supseteq I \Delta_{0}+\exp$ with $K(M)<M$ (i.e., $K(M)=K(M, \emptyset)$ is not cofinal in $M)$, then $\mathbb{N}$ is $\Pi_{1}$-definable in $M$. It is worth noting that both facts can be recovered from Corollary 3.6. Firstly, assume that $M$ is an e.c. model of $\Pi_{2}(\mathbb{N})$. Then, $M$ is a $\Sigma_{1}$-closed model of $\Pi_{1}(\mathbb{N})+\exp$ by Proposition 2.2. Since $\Pi_{1}(\mathbb{N})+\exp$ is coded in every nonstandard model of $\Pi_{2}(\mathbb{N})$, it follows from Corollary 3.6 that $\mathbb{N}$ has a $\Pi_{1}$-definition in $M$. Secondly, assume that $M$ is an e.c. model of $T \supseteq$ $I \Delta_{0}+\exp$ with $K(M)<M$. Again by Proposition 2.2, $M$ is a $\Sigma_{1}$-closed model of $\Pi_{1}(M)+$ exp; and it follows from $K(M)<M$ that $\Pi_{1}(M)+\exp$ is coded in $M$. Thus, by Corollary 3.6, $\mathbb{N}$ is $\Pi_{1}$-definable in $M$.

REMARK 3.8. In Theorem 2.4 of [11] Macintyre and Simmons proved $\mathbb{N}$ to be (parameter free) $\Sigma_{2}$-definable in every $\Sigma_{1}$-closed model of $T \supseteq \Pi_{2}(P A)$. Corollary 3.6 improves this result to $\Pi_{1}$-definability at the price of assuming $T$ to be coded in the model. A natural question then arises: can this assumption be avoided? (I.e., does $\mathbb{N}$ have a $\Pi_{1}$-definition in every $\Sigma_{1}$-closed model of $T \supseteq I \Delta_{0}+\exp$ ?) Let us observe that the answer to this question is negative. To see this, consider a 
recursive binary tree the paths of which correspond to 1-complete extensions of $I \Delta_{0}+$ exp. Then, by the Low Basis Theorem, there is $T_{\text {low }} \subseteq \Sigma_{1} \cup \Pi_{1}$ which is a 1 -complete extension of $I \Delta_{0}+\exp$ of low degree. Take $M \models T_{\text {low }}$. Firstly, it is easy to see that $K(M)$ is a $\Sigma_{1}$-closed model of $T_{\text {low }}$. In addition, $K(M)$ must be nonstandard, for otherwise $\Pi_{1}(\mathbb{N})$ would be recursive in $T_{\text {low }}$. Now suppose that $\theta_{\mathbb{N}}(x) \in \Pi_{1}$ defines $\mathbb{N}$ in $K(M)$. (Note that we can eliminate the possible parameters in $\theta_{\mathbb{N}}(x)$ by using a $\Sigma_{1}$ definition of them.) By $\Delta_{0}$-overspill in $K(M)$, for each $\varphi(x) \in \Delta_{0}$,

$$
\mathbb{N} \models \forall x \varphi(x) \Leftrightarrow K(M) \models \exists x\left(\neg \theta_{\mathbb{N}}(x) \wedge \forall y \leq x \varphi(y)\right) .
$$

Hence, $\Pi_{1}(\mathbb{N})$ would be recursive in $\Sigma_{1}(K(M))$ and so in $T_{\text {low }}$ as well, which is impossible.

We close this section by showing that Theorem 3.4 is best possible with respect to the quantifier complexity of a definition of $\mathbb{N}$ (an alternative model theoretic proof can be given reasoning as in Theorem 8.30 of [7]).

Proposition 3.9. Suppose $I \Delta_{0}+\exp \subseteq T$. Let $M \models T$ be $\Sigma_{1}-$ closed such that $T$ is coded in $M$. Then $\mathbb{N}$ has no $\Sigma_{1}$-definition in $M$.

Proof. Towards a contradiction, assume that $\theta(x, a) \in \Sigma_{1}$ defines $\mathbb{N}$ in $M$. Let $b$ be a code for $T$ in $M$. It follows from Proposition 2.2 that for any $\Pi_{1}$-formula, $\varphi(x, a, b)$, and any element $c \in M$ :

$$
\begin{aligned}
M \models \varphi(c, a, b) \Leftrightarrow & \text { There is } \delta(v, u, z) \in \Sigma_{1} \text { such that } \\
& M \models \delta(c, a, b) \text { and } T \vdash \forall v \forall u \forall z(\delta(v, u, z) \rightarrow \varphi(v, u, z)) \\
\Leftrightarrow & M \models \exists \tau \in \Sigma_{1} \exists y\left\{\begin{array}{l}
\theta(\tau, a) \wedge \theta(y, a) \wedge \operatorname{Tr}_{1}(\tau(\tilde{c}, \tilde{a}, \tilde{b})) \wedge \\
\operatorname{Prf}_{T}(\forall v \forall u \forall z(\tau(v, u, z) \rightarrow \varphi(v, u, z)), y, b),
\end{array}\right.
\end{aligned}
$$

where $\operatorname{Prf}_{T}(w, y, b)$ expresses " $y$ encodes a proof of a formula $w$ in $T$ " (we use the parameter $b$ to express the set of axioms of $T)$. Hence, $\Pi_{1}$ truth of $(M, a, b)$ turns out to be $\Sigma_{1}$-definable in $(M, a, b)$, which contradicts Tarski's theorem.

Since the recursive sets are coded in every nonstandard model of $I \Delta_{0}$, we get

Corollary 3.10. Suppose $T$ is a recursive extension of $I \Delta_{0}+\exp$. Then, $\mathbb{N}$ does not have a $\Sigma_{1}$-definition in any $\Sigma_{1}$-closed model of $T$.

4. Recursive saturation and distribution of definable elements. In this section we determine the precise amount of recursive saturation available in each $\Sigma_{1}$-closed model of $I \Delta_{0}+\exp$ and derive some applications. First of all, recall that a model $M$ satisfies $\Sigma_{1}$-overspill for the standard cut if for every $\varphi(x, a) \in \Sigma_{1}$,

$$
\forall k \in \omega, M \models \varphi(k, a) \Rightarrow \exists b>\omega, M \models \forall x \leq b \varphi(x, a) .
$$

Proposition 4.1. Suppose $I \Delta_{0}+\exp \subseteq T$. Let $M \models T$ be $\Sigma_{1}-$ closed such that $T$ is coded in $M$. Then, $M$ has $\Sigma_{1}$-overspill for the standard cut.

Proof. Consider $\varphi(x, a) \in \Sigma_{1}$ such that $M \models \varphi(k, a)$ for all standard $k$. Put $\varphi(x, v) \equiv \exists y \varphi_{0}(x, y, v)$, with $\varphi_{0} \in \Delta_{0}$. Then, $\psi(k) \equiv \exists u \forall x \leq k \exists y \leq u \varphi_{0}(x, y, a)$ is true in $M$ for all standard $k$. Since $\mathbb{N}$ has no $\Sigma_{1}$-definition in $M$ (Proposition 3.9), there must be some $b>\omega$ such that $M \models \psi(b)$ and the result follows.

In [16], Simmons proved Proposition 4.1 for each $\Sigma_{1}$-closed model of $T \supseteq I \Delta_{0}+\exp$ in which the $\Sigma_{1}$ definable elements are not cofinal, and asked whether 
this assumption could be eliminated (Problem 6.5 of [16]). Notice that Proposition 4.1 answers in the positive this question if we restrict ourselves to recursively axiomatized theories, as the recursive sets are always coded. In contrast, the answer to Simmons's question for $T$ arbitrary is negative. To see this, consider $M \models I \Delta_{0}+\exp$ with $a \in M$ nonstandard and $\Delta_{0}$-definable. As usual, $2_{y}^{x}$ stands for the function $2_{0}^{x}=$ $x ; 2_{y+1}^{x}=2^{2_{y}^{x}}$ (cf. [6]). Let $I$ be the initial segment of $M$ given by $\left\{b \in M: b<2_{n}^{a}\right.$ for some $n \in \mathbb{N}\}$ and put $N=K(I)$. (Note that $a$ is in $N$ since $a$ is $\Delta_{0}$-definable.) Then, $N$ is easily seen to be a $\Sigma_{1}$-closed model of $\Pi_{1}(N)+\exp$. But the $\Sigma_{1}$ formula $\exists z\left(z=2_{y}^{a}\right)$ defines $\mathbb{N}$ in $N$ and thus $N$ does not have $\Sigma_{1}$-overspill for $\mathbb{N}$.

Observe that the previous argument shows that Simmons's question has a negative answer already for $\Delta_{2}$ definable theories. It suffices to justify that $\Pi_{1}(N)$ can be taken $\Delta_{2}$ definable. Take $S=I \Delta_{0}+\exp +\neg \varphi$ consistent, with $\varphi \in \Pi_{1}(\mathbb{N})$. Take $T^{*} \subseteq \Pi_{1}$ recursive in $0^{\prime}$ (and so $\Delta_{2}$ definable) and maximally consistent with $S$, i.e. there is no $\Pi_{1}$ sentence consistent with $S+T^{*}$ which is not in $T^{*}$. For the existence of such a set, fix a recursive enumeration of $\Pi_{1}$-sentences and consider the leftmost path of a recursive binary tree the paths of which correspond to 1-complete extensions of $S$, see the proof of Theorem 5.2 for a similar construction. Now start with $M \models S+T^{*}$ and define $I$ and $N=K(I)$ as before (note that $M$ contains nonstandard $\Delta_{0}$ definable elements by $\left.\neg \varphi\right)$. Then, $N \models S+T^{*}$ and so $\Pi_{1}(N)=T^{*}$ by the maximality condition for $T^{*}$.

Putting together overspill and the ability of coding automatically gives some amount of recursive saturation in a model of arithmetic. In fact, we have

Proposition 4.2. Suppose I $\Delta_{0}+\exp \subseteq T$. Let $M \models T$ be $\Sigma_{1}-$ closed with $T$ coded in $M$. Then, $M$ is $\Sigma_{1}$-recursively saturated and not short $\Pi_{1}-$ recursively saturated.

Proof. By Corollary 3.6, there is $\theta_{T}(x, a) \in \Pi_{1}$ defining $\mathbb{N}$ in $M$. Hence, $M$ is not short $\Pi_{1}$-recursively saturated; the type over $M$

$$
\{x<b\} \cup\left\{x>n \wedge \theta_{T}(x, a): n \in \omega\right\}
$$

is omitted, where $b$ is any nonstandard element. In order to prove that $M$ is $\Sigma_{1}$-recursively saturated, let $p(v, a)$ be a recursive $\Sigma_{1}$ type over $M$ and let $b \in M$ be a code of $p(v, a)$ in $M$. Since $B \Sigma_{1}+\exp$ is $\Pi_{2}$ conservative over $I \Delta_{0}+\exp$, there exists $N \models B \Sigma_{1}+\exp$ satisfying that $M \prec_{1} N$. Put

$$
\varphi(x, z, a, b) \equiv \forall i \leq z\left((b)_{i} \neq 0 \rightarrow \operatorname{Sat}_{1}(i, x, a)\right),
$$

where $\operatorname{Sat}_{1}(i, x, v)$ denotes the usual satisfaction predicate for $\Sigma_{1}$-formulas. Then, for each $n \in \omega, N \models \exists x \varphi(x, n, a, b)$. Consider $\varphi^{\prime}(x, z, a, b) \in \Sigma_{1}$ equivalent in $B \Sigma_{1}$ to $\varphi(x, z, a, b)$. Then, $M \models \exists x \varphi^{\prime}(x, n, a, b)$ for each $n \in \omega$. Since $M$ satisfies $\Sigma_{1}$-overspill for $\mathbb{N}$, there must be some $c \in M$ nonstandard and $d \in M$ such that $M \models \varphi^{\prime}(d, c, a, b)$ and hence $N \models \varphi(d, c, a, b)$. It is easy to see that such an element $d$ realizes $p(v, a)$ in $M$.

Let us observe that the use of $B \Sigma_{1}$ in the previous argument is inessential. As suggested by the anonymous referee, one could avoid appealing to the $\Pi_{2}$-conservation of $B \Sigma_{1}+\exp$ over $I \Delta_{0}+\exp$ by assuming that the type $p(v, a)$ is enumerated as $\left\{\theta_{i}(v, a): i \in \omega\right\}$, in which each $\theta_{i+1}(v, a)$ is a $\Sigma_{1}$-formula of the form $\theta_{i}(v, a) \wedge \eta_{i}(v, a)$ for some $\eta_{i} \in \Sigma_{1}$. 
We say that a model $M$ is regular (or $\Sigma_{1}-$ tall in Lessan's terminology, cf. [10]) if for every $a \in M, K(M, a)$ is not cofinal in $M$. Regular $\Sigma_{1}$-closed models have nice properties, especially in connection with immersion theorems à la Friedman (see [7] or [16]). In [16] Simmons introduced the notion of a well built model in order to isolate the most important features of regular $\Sigma_{1}$-closed models. Namely, $M$ is well built if $M$ is $\Sigma_{1}$-closed and

(a) $M$ satisfies $\Sigma_{1}$-overspill for the standard cut,

(b) $\operatorname{Def}_{\Sigma_{1}}(M) \cap \mathbb{N} \subseteq S S y(M)$, i.e., the standard part of each $\Sigma_{1}$-definable subset of $M$ is coded in $M$.

Simmons asked for exhibiting a countable well built model which is not regular (see Problem 6.15 in [16]). However, our next result shows that both notions coincide. We are able to prove even a stronger result: condition (b) alone is equivalent to regularity.

Proposition 4.3. Suppose $I \Delta_{0}+\exp \subseteq T$. Let $M \models T$ be $\Sigma_{1}-$ closed. The following are equivalent.

1. $M$ is regular.

2. $\operatorname{Def}_{\Sigma_{1}}(M) \cap \mathbb{N} \subseteq S S y(M)$.

3. For all $a \in M$, the $\Sigma_{1}$-type of $a$ in $M, t p_{\Sigma_{1}}^{M}(a)$, is coded in $M$.

Proof. $(1 \Rightarrow 2)$ : Consider $\varphi(x, v) \in \Sigma_{1}$ and $a \in M$. Put $\varphi(x, v)=\exists y \varphi_{0}(x, y, v)$, with $\varphi_{0} \in \Delta_{0}$, and suppose that $K(M, a)$ is bounded by $b$. Then, for each $n \in \omega$, $M \models \varphi(n, a) \leftrightarrow \exists y \leq b \varphi_{0}(n, y, a)$ and so $\{n \in \mathbb{N}: M \models \varphi(n, a)\}$ is coded in $M$. $(2 \Rightarrow 3)$ : Immediate.

$(3 \Rightarrow 1)$ : By part $(3), \Sigma_{1}(M)$ is coded in $M$ and hence so is $\Pi_{1}(M)+$ exp. By Proposition 2.2, $M$ is a $\Sigma_{1}-$ closed model of $\Pi_{1}(M)+\exp$. Then, $M$ is $\Sigma_{1}-$ recursively saturated by Proposition 4.2. Now pick $a \in M$. Suppose that $b \operatorname{codes} t p_{\Sigma_{1}}^{M}(a)$ in $M$

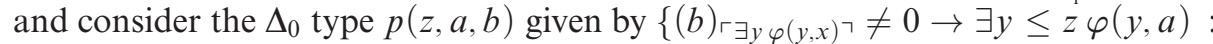
$\left.\varphi(x, v) \in \Delta_{0}\right\}$. By recursive saturation, there is $c \in M$ realizing $p(z, a, b)$ in $M$. It is clear that $K(M, a)$ is bounded by $c$.

A model $M$ is simple if $M=K(M, a)$ for some $a \in M$. Clearly, simple $\Sigma_{1}$-closed models are examples of nonregular $\Sigma_{1}-$ closed models. In [7] Hirschfeld raised the question whether some other examples could exist. That is to say, can a $\Sigma_{1}$-closed model be neither regular nor simple? To the best of our knowledge, the answer to this question is still unknown. Here we have only obtained the following related result.

Proposition 4.4. Suppose $I \Delta_{0} \subseteq T$. Let $M \models T$ be $\Sigma_{1}$-closed.

1. Every $\Sigma_{2}$-definable element of $M$ is also $\Sigma_{1}$-definable.

2. If $T$ is recursive, there is $a \in K(M)$ with $K(M,(\leq a))$ cofinal in $M$.

3. If $T \vdash \exp$, for every nonstandard $a \in K(M), M=K(M,(\leq a))$.

Proof. (1) Let $a \in M$ and let $\delta(x) \in \Sigma_{2}$ such that $M \models \delta(a) \wedge \forall x(\delta(x) \rightarrow$ $x=a)$. Put $\delta(x) \equiv \exists y \delta_{0}(x, y)$ with $\delta_{0}(x, y) \in \Pi_{1}$. Then there exists $b \in M$ such that $M \models \delta_{0}(a, b)$. Since $\delta_{0}(x, y) \in \Pi_{1}$ and $M$ is $\Sigma_{1}$-closed, there exists $\theta(x, y) \in \Sigma_{1}$ such that $M \models \theta(a, b)$ and $T \vdash \forall x \forall y\left(\theta(x, y) \rightarrow \delta_{0}(x, y)\right)$. As a consequence, $K(M) \models \exists x \exists y \theta(x, y)$, since $K(M) \prec_{1} M$. So there are $c, d \in K(M)$ such that $M \models \theta(c, d)$. Thus, we get $M \models \delta_{0}(c, d)$ and it follows that $M \models \delta(c)$. Hence, $a=c \in K(M)$. 
(2) For the sake of a contradiction assume that for all element $a \in K(M)$, $K(M,(\leq a))$ is bounded in $M$. We shall prove that $M \models L \Sigma_{1}^{-}$(least number principle for parameter free $\Sigma_{1}$-formulas). Since $L \Sigma_{1}^{-}$and $I \Pi_{1}^{-}$are well known to be equivalent over $I \Delta_{0}$, this would contradict Proposition 2.4. So let $\varphi(x, y) \in \Delta_{0}$ such that $M \models \exists x \exists y \varphi(x, y)$. Pick $a \in K(M)$ such that $M \models \exists y \varphi(a, y)$. By hypothesis, $K(M,(\leq a))$ is bounded in $M$ by some $b \in M$. Then, for all $d \leq a$,

$$
M \models \exists y \varphi(d, y) \Longleftrightarrow K(M,(\leq a)) \models \exists y \varphi(d, y) \Longleftrightarrow M \models \exists y<b \varphi(d, y) .
$$

By $I \Delta_{0}$, there exists $c=\min \{d \in M: M \models d \leq a \wedge \exists y<b \varphi(d, y)\}$. Then, in $M, c=(\mu x)(\exists y \varphi(x, y))$, as required.

(3) Let $\theta(a)$ denote the $\Pi_{2}$ formula $\forall x \exists y \exists z \leq a\left(\operatorname{Min}_{0}(z, y) \wedge x=(y)_{0}\right)$, where $\operatorname{Min}_{0}(z, y)$ is a $\Sigma_{1}$-formula expressing (in $I \Delta_{0}+\exp$ ) that " $y$ is the least element satisfying the $\Delta_{0}$-formula $z$." Since every $\Sigma_{1}$-definable element of $M$ can be obtained as a projection of a $\Delta_{0}$-minimal element, we obtain that, for every nonstandard $a \in K(M), K(M) \models \theta(a)$. Therefore, $M \models \theta(a)$ (recall that $K(M) \prec_{2} M$ ). Thus, given $b \in M$, there are $p \leq a$ and a (unique) $c \in M$ such that $M \models$ $\operatorname{Min}_{0}(p, c) \wedge b=(c)_{0}$. So, $b \in K(M,(\leq a))$, as required.

5. Turing degrees of maximal theories. Let $S$ be a recursive theory. We say that a theory $T^{\star}$ consisting of $\Sigma_{1}$ sentences is maximal with respect to (w.r.t.) $S$ if $T^{\star}$ is maximal consistent with $S$, i.e., there is no $\Sigma_{1}$ sentence consistent with $S+T^{\star}$ which is not already in $T^{\star}$. Maximal theories and $\Sigma_{1}$-closed models are tightly connected. (Actually, Lemma 5 of [4] shows that $T^{\star} \subseteq \Sigma_{1}$ is maximal w.r.t. $S$ if, and only if, there exists some $M=\Pi_{2}(S) \Sigma_{1}$-closed with $\Sigma_{1}(M)=T^{\star}$.) In studying the complexity of a maximal theory from the point of view of the Turing degrees, the first author proved that:

THEOREM 5.1 ([1]). Let $S$ be a recursive extension of $I \Delta_{0}+$ exp. Then $0^{\prime} \leq_{T} T^{\star}$ for every theory $T^{\star}$ which is maximal w.r.t. $S$.

Here we observe that Theorem 5.1 can also be obtained as a direct consequence of the existence of $\Pi_{1}$-definitions of the standard cut in $\Sigma_{1}$-closed models.

Proof of Theorem 5.1. Let $T^{\star}$ be maximal w.r.t. $S$. We shall identify $0^{\prime}$ with $\Pi_{1}(\mathbb{N})$. Let $M$ be a model of $S+T^{\star}$. It is easy to see that the submodel of $\Sigma_{1}-$ definable elements $K(M)$ is a $\Sigma_{1}$-closed model of $\Pi_{2}(S)$. It then follows from Corollary 3.5 that there exists $\theta(x) \in \Pi_{1}^{-}$defining $\mathbb{N}$ in $K(M)$. By an overspill argument, for each $\varphi(x) \in \Delta_{0}$

$$
\mathbb{N} \models \forall x \varphi(x) \Longleftrightarrow K(M) \models \exists y(\neg \theta(y) \wedge \forall x \leq y \varphi(x)) .
$$

Hence, $\Pi_{1}(\mathbb{N}) \leq_{T} \Sigma_{1}(K(M))=T^{\star}$.

Theorem 5.1 is best possible in that for each recursive theory $S \supseteq I \Delta_{0}+\exp$ there is $T^{\star}$ which is maximal w.r.t. $S$ and of Turing degree $0^{\prime}$. (Take the leftmost path of an appropriate recursive binary tree, see the proof of Theorem 5.2 below for details.) On the other hand, it is natural to ask: is every Turing degree above $0^{\prime}$ the degree of a maximal theory? The next result gives a positive answer to this question.

THEOREM 5.2. Let $S$ be a recursive extension of $I \Delta_{0}+\exp$ and let $A \subseteq \mathbb{N}$ such that $0^{\prime} \leq_{T}$ A. Then, there exists $T^{\star}$ which is maximal w.r.t. $S$ and such that $A \equiv_{T} T^{\star}$. 
Proof. Since $0^{\prime} \leq_{T} A$, it follows by the Jump Inversion Theorem that the degree of $A$ is the jump of a degree realizing the least possible jump, i.e., there exists $B \subseteq \mathbb{N}$ satisfying that $A \equiv_{T} B^{\prime} \equiv_{T} B \oplus 0^{\prime}$ (for a proof see, e.g., Theorem V.2.24 of [13]). We first show that there is $\delta(x) \in \Sigma_{1}$ such that

- $I \Delta_{0} \vdash \forall x, y(\delta(x) \wedge \delta(y) \rightarrow x=y)$, and

- the theory $S_{B}$ given by

$$
S+\left\{\exists x\left(\delta(x) \wedge(x)_{i} \neq 0\right): i \in B\right\}+\left\{\exists x\left(\delta(x) \wedge(x)_{i}=0\right): i \notin B\right\}
$$

is consistent.

To this end, we adapt the proof of Proposition 4.2.6 in [10], where it is shown that every $X \subseteq \mathbb{N}$ can be coded by a $\Sigma_{1}$ definable element in some model of $P A$. Pick $\varphi \in \Pi_{1}(\mathbb{N})$ such that $S^{\prime}=\Pi_{1}(S)+I \Sigma_{1}+\neg \varphi$ is consistent. Let $\theta(x) \in \Sigma_{1}$ be a flexible formula w.r.t. $S^{\prime}$; that is to say, $\theta(x)$ has the property that if we choose arbitrarily $\theta(n)$ or $\neg \theta(n)$, but not both, for every numeral $n$, then this set of sentences together with $S^{\prime}$ is consistent for every such choice. (See Theorem III-2.15 of [6] for a proof of the existence of flexible formulas.) In particular, the theory given by $S^{\prime}$ augmented with

$$
\begin{gathered}
\{\theta(2 n): n \in B\}+\{\neg \theta(2 n): n \notin B\}+ \\
\{\theta(2 n+1): n \notin B\}+\{\neg \theta(2 n+1): n \in B\}
\end{gathered}
$$

is consistent and so has a model, say $M$. Note that $K(M)$ contains nonstandard elements since $\neg \varphi$ is true in $M$. Pick $a \in K(M)$ nonstandard. By applying $I \Sigma_{1}$ in $M$ it follows that

$$
M \models \exists x \forall i \leq a\left[\left((x)_{i} \neq 0 \rightarrow \theta(2 i)\right) \wedge\left((x)_{i}=0 \rightarrow \theta(2 i+1)\right)\right] .
$$

Since $K(M) \prec_{1} M$, there exists $b \in K(M)$ such that

$$
M \models \forall i \leq a\left[\left((b)_{i} \neq 0 \rightarrow \theta(2 i)\right) \wedge\left((b)_{i}=0 \rightarrow \theta(2 i+1)\right)\right]
$$

and hence $b$ codes $B$ in $M$. Since $M$ satisfies $\Pi_{1}(S)$, there is $N \models S$ satisfying that $M \prec_{0} N$. Clearly, $b$ codes $B$ in $N$ too. Let $\exists z \delta^{\prime}(x, z)$ be a $\Sigma_{1}$ definition of $b$ in $M$, with $\delta^{\prime}(x, z) \in \Delta_{0}$. It is easy to see that $\delta(x)=\exists u\left(x=(u)_{0} \wedge u=\mu t . \delta^{\prime}\left((t)_{0},(t)_{1}\right)\right)$ satisfies the required properties.

Now we extend $S_{B}$ to a maximal theory by taking the leftmost path of a binary tree. Fix a recursive enumeration of $\Sigma_{1}$ sentences, $\varphi_{1}, \varphi_{2}, \ldots$ We put

$$
\sigma \in C \Leftrightarrow \begin{aligned}
& S_{B}+\left\{\varphi_{i}: \sigma(i)=0\right\}+\left\{\neg \varphi_{i}: \sigma(i)=1\right\} \\
& \text { does not prove a contradiction with proof } \leq \operatorname{length}(\sigma)^{\circ} .
\end{aligned}
$$

Then $C$ is a binary tree recursive in $B$. Let $f: \mathbb{N} \rightarrow\{0,1\}$ be the leftmost path of $C$ and put $T^{*}=\left\{\varphi_{i}: f(i)=0\right\}$. Then, we have

- $T^{*}$ is maximal w.r.t. $S$ by construction.

- $T^{*} \leq_{T} A$. Being determined by the leftmost path of the binary tree $C, T^{*}$ is recursive in $C^{\prime}$ and hence $T^{*} \leq_{T} B^{\prime} \equiv_{T} A$.

- $A \leq_{T} T^{*}$. First, $B \leq_{T} T^{*}$ since $i \in B \Leftrightarrow \exists x\left(\delta(x) \wedge(x)_{i} \neq 0\right) \in T^{*}$. Second, $0^{\prime} \leq_{T} T^{*}$ by Theorem 5.1. Thus, $A \equiv_{T} B \oplus 0^{\prime} \leq_{T} T^{*}$.

This completes the proof. 
Concerning Scott sets, it is well known that every countable Scott set closed under arithmetical definability is the standard system of a regular $\Sigma_{1}$-closed model of $I \Delta_{0}+\exp$ (see [7] or [16]). In addition, it is a result of McAloon (see Theorem 5.1 of [12]) that every countable model of $P A$ has an end extension which is $\Sigma_{1}$-closed w.r.t. $\Pi_{2}(P A)$. Since the standard system is preserved by end extensions, it then follows that every countable Scott set is the standard system of a $\Sigma_{1}$-closed model of $\Pi_{2}(P A)$. A natural question arises: Is every countable Scott set the standard system of a $\Sigma_{1}-$ closed model of $I \Delta_{0}+\exp$ ?

6. Acknowledgment. We thank the anonymous referee for his/her helpful comments and suggestions. The first author is grateful to Wojciech Moczydłowski who first pointed to her the gap in the proof of Corollary 1.3 of [2]. The first author was supported in part by grant NCN nr 2013/09/B/ST1/04390. The second and third authors were supported in part by Spanish Government grant MTM2011-26840.

\section{REFERENCES}

[1] Z. Adamowicz, On maximal theories, this Journal, vol. 56 (1991), pp. 885-890.

[2] Z. AdAmowicz and T. BigorajSKa, Existentially closed structures and Gödel's second incompleteness theorem, this JournAL, vol. 66 (2001), pp. 349-356.

[3] Z. Adamowicz, L. A. KołodzIEJCZYK, and J.B. Paris, Truth definitions without exponentiation and the $\Sigma_{1}$ collection scheme, this JournaL, vol. 77 (2012), pp. 649-655.

[4] A. Cordón-Franco, A. Fernández-Margarit, and F. F. Lara-Martín, A note on $\Sigma_{1}$-maximal models, this JournaL, vol. 72 (2007), pp. 1072-1078.

[5] D. C. Goldrei, A. Macintyre, and H. Simmons, The forcing companions of number theories. Israel Journal of Mathematics, vol. 14 (1973), pp. 317-337.

[6] P. HÁJEK and P. PudLÁK, Metamathematics of First-order Arithmetic, Perspectives in Mathematical Logic, Springer-Verlag, Berlin, 1993.

[7] J. HirschFeld and W. WheELER, Forcing, Arithmetic, Division rings, Lecture Notes in Mathematics, vol. 454, Springer-Verlag, Berlin, 1975.

[8] R. KaYe, Models of Peano Arithmetic, Oxford Logic Guides, vol. 15, Oxford University Press, Oxford, 1991.

[9] H. Kotlarski, An addition to Rosser's theorem, this Journal, vol. 61 (1996), pp. 285-292.

[10] H. Lessan, Models of Arithmetic, Ph.D. thesis, University of Manchester, 1978.

[11] A. Macintyre and H. Simmons, Algebraic properties of number theories. Israel Journal of Mathematics, vol. 22 (1975), pp. 7-27.

[12] K. McAloon, Completeness theorems, incompleteness theorems and models of arithmetic. Transactions of the American Mathematical Society, vol. 239 (1978), pp. 253-277.

[13] P. G. Odifredd, Classical Recursion Theory, Studies in Logic and the Foundations of Mathematics, vol. 125, North-Holland, Amsterdam, 1989.

[14] A. Robinson, Non standard arithmetic and generic arithmetic, Logic, Methodology and Philosophy of Science IV (P. Suppes et al., editors), North-Holland, Amsterdam, 1974, pp. 137-154.

[15] H. Simmons, Existentially closed structures, this JournaL, vol. (1972), pp. 293-310.

[16] - Existentially closed models of basic number theory, Logic Colloquium 76 (R. Gandy and M. Hyland, editors), Studies in Logic and the Foundations of Mathematics, vol. 87, North-Holland, Amsterdam, 1977, pp. 325-369.

[17] A. J. WILKIE and J. B. PARIS, On the scheme of induction for bounded arithmetic formulas. Annals of Pure and Applied Logic, vol. 35 (1987), pp. 261-302. 